\title{
Design of an access control mechanism for the Available Bit Rate service in ATM networks
}

\author{
F. Bernabei * , G. Chierchia * , L. Gratta *, M. Listanti \# \\ * Fondazione Ugo Bordoni \\ via B. Castiglione 59 - 00142 Roma (Italy); +39 65480 3410; \\ bernabei@fub.it; laura@fub.it \\ \# INFOCOM Dept., University "La Sapienza" of Roma \\ via Eudossiana 18 - 00184 Roma (Italy); +396 44585458; \\ marco@infocom.ing.uniromal.it
}

\begin{abstract}
In this paper a mechanism for the control of the Available Bit Rate traffic at the access of ATM networks is presented. It is based on the integration of the backpressure and a credit based mechanism. A credit distribution policy among sources, aiming at equalizing the occupancy of the transmission buffers of the sources, is defined. Moreover, the introduction of an uncontrolled mode of operation when the network status is far from congestion allows the signaling overhead to be limited as in the backpressure scheme. The proposed control scheme allows the achievement of very good performance in terms of cell loss and a low signaling overhead. A performance study of the proposed mechanism is presented. Various traffic scenarios are considered. Results arising from simulation are compared with performance of the backpressure mechanism. Moreover, analytical bounds for the cell loss probability are found and compared with the performance derived from simulation.
\end{abstract}

\section{Keywords}

Available Bit Rate service, congestion control, credit mechanism, backpressure.

\section{INTRODUCTION}

The Asynchronous Transfer Mode (ATM) provides a substrate for network resource sharing among various service classes. A strong activity, in stardardization bodies and ATM Forum, aiming at defining how to use this ATM substrate is now in progress. The basic guideline is the definition of two main service classes: Guaranteed Bandwidth (GB) services and Available Bit Rate (ABR) services. The ABR communications do not reserve any buffer or bandwidth resource, but share a common network capacity made up of the bandwidth unused by GB

\footnotetext{
Work carried out in the framework of the agreement between Fondazione Ugo Bordoni and the Italian PT Administration, and partially funded by the RACE Projrct 2068 (LACE).
} 
connections. The Quality of Service (QoS) perceived by ABR service users highly depends on the network status. The ABR service is considered to be the key service for the ATM LAN.

Various proposals are available in literature for the ABR traffic control in ATM networks [1, $2,3,4]$. Their goal is to avoid cell loss inside the ATM network, constraining it at the sources. To this aim, the backpressure mechanism proposed in $[3,4]$ uses stop and go signals to control the traffic emitted by sources. One of the main advantages of the backpressure is that in case of low traffic no signalling is required. Though very simple, this method becomes unapplicable as soon as the network latency grows. More flexible schemes are based on credit mechanisms [2]. According to these schemes, the number of cells that a source can emit is explicitly communicated to the source. At least in principle, this class of mechanisms does not suffer any distance limitation and can work in any delay condition.

In this paper, a mechanism is proposed, which is an integration of the above schemes. In our approach we have held the backpressure property of no signaling for low traffic, introducing two operation modes: the uncontrolled and the controlled one. In the uncontrolled mode (buffer occupancy remains under a given threshold) the sources send cells at the peak bit rate. If the mentioned threshold is exceeded, the multiplexer enters in the controlled mode, during which the trasmission by the sources is regulated by means of credits. Differently from the backpressure, according to which only two trasmission states are possible (source peak rate or complete block), according to this mechanism trasmission can occur at the source peak rate or can be regulated by credits.

The basic aspects of the proposed control mechanism are:

- two modes of operation: uncontrolled and controlled;

- credit requests and distribution are performed according to periodic signaling cycles;

- the number of credits assigned by the Mux is evaluated, cycle by cycle, through a prediction of the sources status;

- the distribution of credits among sources has been defined aiming at equalizing the occupancy of the transmission buffers.

The proposed mechanism can be conveniently implemented at the ATM network access. We assume that the access multiplexer is fed by loss sources representing for example bridges between LANs and an ATM network. In this case, the traffic offered to the LAN (and consequently to the bridge) cannot be controlled on the basis of the ATM network status.

This paper gives a detailed description of the credit-based ABR traffic control mechanism (Sec. 2) and of the adopted credit distribution strategy (Sec. 3). In Sec. 4, a performance study of the proposed mechanism is presented. Results arising from simulation are compared with performance of the backpressure mechanism.

\section{CREDIT-BASED MECHANISM DESCRIPTION}

The considered access scenario is shown in the Figure 1. A number $N$ of ABR Traffic Sources (TSs) are connected through dedicated links to an access multiplexer (Mux). A Transmission Buffer (TB) is associated to each source, this buffer is utilized to store the cells blocked by the traffic control mechanism. A traffic source together with the associated transmission buffer forms a Controlled Source (CS).

The Mux output capacity is shared between ABR and GB traffic. The ABR traffic grabs the portion of output capacity of the Mux unused by the GB traffic.

Let us introduce some notations:

$B_{\text {mux }}$ Mux buffer size (in cells);

$B_{i} \quad$ size (in cells) of the transmission buffer of the $i$-th controlled source $(1 \leq i \leq N)$;

$F_{i} \quad$ peak bit rate of the $i$-th traffic source $(1 \leq i \leq N)$;

$A_{i} \quad$ average bit rate of the $i$-th traffic source $(1 \leq i \leq N)$;

$P_{i} \quad$ peak bit rate of the controlled source; we assume that $P_{i} \geq F_{i},(1 \leq i \leq N)$;

$\tau_{\max }$ maximum round trip delay between CSs and the Mux (in $\mu \mathrm{s}$ );

$L_{c} \quad$ cell size (in bit). 


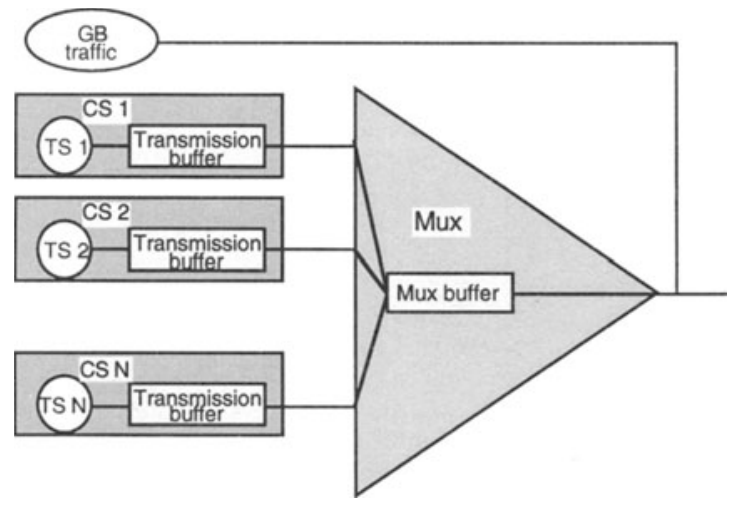

Figure 1 - Access scenario.

The ABR traffic control mechanism, here considered, is based on two operation modes: uncontrolled and controlled. The transition between these two modes depends on the occupancy level of the Mux buffer: if it is scarcely utilized, the mechanism is in the uncontrolled mode; when a buffer congestion occurs, i.e. as soon as the buffer occupancy exceeds an activation threshold $T_{a}$, the system passes to the controlled mode. This mode is maintained until the Mux buffer occupancy goes down a deactivation threshold $T_{d}$.

If the uncontrolled mode is active, the $i$-th CS emits its cells at the source peak bit rate $F_{i}$. The value of the activation threshold $T_{a}$ can be determined by considering the worst case of cell arrival at the Mux with the constraint that cell loss has to be avoided. The activation threshold $T_{a}$ is given by:

$T_{a}=\mathrm{B}_{\operatorname{mux}}-\sum_{\mathrm{i}=1}^{\mathrm{N}}\left\lceil\frac{\mathrm{F}_{\mathrm{i}}}{\mathrm{L}_{\mathrm{c}}} \tau_{\max }\right\rceil$

wherein $\lceil x\rceil$ represents the ceiling function of $x$. The evaluation of $T_{a}$ is carried out considering the maximum round trip delay $\tau_{\text {max }}$ in order not to advantage those CSs nearest to the multiplexer. If the evaluated $\mathrm{T}_{\mathrm{a}}<0$ the system is always in the controlled mode.

The operation of the traffic control mechanism in the controlled mode is based on the following rules:

1. as soon as the occupancy of the Mux exceeds $T_{a}$, the Mux sends to every CS an activation message indicating the beginning of the controlled mode;

2. each CS periodically estimates the future occupancy of its transmission buffer and communicates it to the Mux (forward signaling);

3. the Mux periodically communicates to each CS the number of assigned credits (backward signaling); the total amount of assigned credits is determined avoiding cell loss at Mux; the distribution of credits among CSs aims at minimizing the global cell loss at CSs.

4. during the controlled mode, the cell emission by CSs takes place at the peak rate $P_{i}$ and is regulated by credits provided by the Mux; one credit enables a CS to emit a single cell;

The Figure 2 shows the time diagram relevant to a generic forward-backward signaling exchange in the controlled mode. To simplify the explanation of the procedures, we consider, without loss of generality, that all the CSs are at the same distance from the Mux. This implies that the time diagram of the Figure 2 holds for all the CSs. It can be straightforwardly argued that if the distances CS-Mux were different, the traffic control mechanism behaves as if all the CSs were at the same distance from the Mux, equal to the maximum one. 


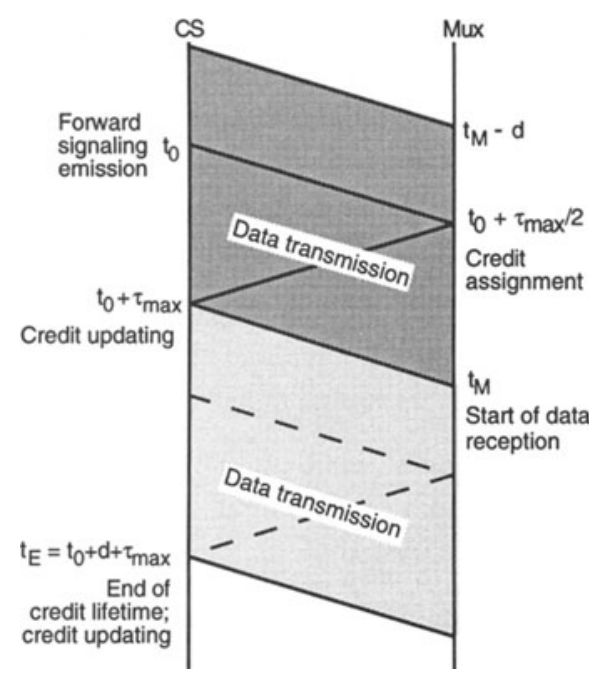

Figure 2-Signaling exchange time diagram.
Let $t_{0}$ be the time instant in which the source sends its forward signaling and let $d$ be the signaling period, i.e. the time interval elapsing between the emission of two successive forward (backward) signaling messages by a CS (Mux).

The forward signaling carries the estimate of the transmission buffer occupancy at a future time instant $t_{E}=t_{0}+\tau_{\max }+d$.

Several estimate procedures have been tested in our study, however the obtained performance results do not differ substantially, so, the simplest one is here discussed. This procedure corresponds to suppose that, if at time $t_{0}$ a TS is active (i.e. it is emitting a burst of data), it remains active for the whole time interval $\left[t_{0}, t_{E}\right]$. On the contrary, if at time $t_{0}$ the TS is idle, no new cell emission is foreseen in $\left[t_{0}, t_{E}\right]$.

Let $b_{i}\left(t_{0}\right)$ be the occupancy (in cells) of the transmission buffer of the $i$-th CS $(1 \leq i \leq N)$ at time $t_{0}$ and let $a_{i}\left(t_{0}\right)$ be the number of credits still unused by the $i$-th CS

at time $t_{0}$. The estimate $\mathrm{b}_{\mathrm{i}}^{\mathrm{e}}\left(\mathrm{t}_{\mathrm{E}}\right)$ of the buffer occupancy at time $t_{E}$ is done assuming that no credits are assigned to the $i$ th $C S$ at time $t_{0}+\tau_{\max }$, and is given by:

$b_{i}^{e}\left(t_{E}\right)=b_{i}\left(t_{0}\right)-a_{i}\left(t_{0}\right)+e_{i}\left(t_{E}\right)$ $1 \leq \mathrm{i} \leq \mathrm{N}$

wherein $e_{i}\left(t_{E}\right)$ is the estimate of the number of cells originated by the $i$-th TS within the interval $\left[t_{0}, t_{E}\right]$. According to the above mentioned estimate procedure

$e_{i}\left(t_{E}\right)= \begin{cases}0 & \text { if the } i-t h \text { TS is idle at time } t_{0} \\ \left\lceil\frac{\left(t_{E}-t_{0}\right) F_{i}}{L_{c}}\right\rceil & \text { if the } i-t h \text { TS is active at time } t_{0}\end{cases}$

At time $t_{1}=t_{0}+\tau_{\max } / 2$ the Mux evaluates its own buffer occupancy at the target instant $t_{M}=t_{1}+\tau_{\max }$. To avoid losses in the Mux buffer, the worst case occupancy is considered, that occurs if the Mux output capacity is entirely utilized by the GB traffic in $\left[t_{0}, t_{E}\right]$.

Let $b_{\text {mux }}\left(t_{1}\right)$ and $b_{\text {mux }}^{e}\left(t_{M}\right)$ be the Mux buffer occupancy at time $t_{I}$ and the evaluated buffer occupancy at time $t_{M}$, respectively. Moreover, let $a_{m u x}\left(t_{1}\right)$ be the difference between the total amount of credits distributed by the Mux in the last $\left\lceil\tau_{\max } / d\right\rceil$ signaling cycles and the number of received cells relevant to these cycles. It results

$\mathrm{b}_{\text {mux }}^{\mathrm{e}}\left(\mathrm{t}_{\mathrm{M}}\right)=\mathrm{b}_{\text {mux }}\left(\mathrm{t}_{1}\right)+\mathrm{a}_{\text {mux }}\left(\mathrm{t}_{1}\right)$

In order to avoid cell loss at Mux, the global amount $C_{\text {tot }}\left(t_{1}\right)$ of credits that can be assigned at time $t_{l}$ is

$\mathrm{C}_{\text {tot }}\left(\mathrm{t}_{1}\right)=\mathrm{B}_{\text {mux }}-\mathrm{b}_{\text {mux }}^{\mathrm{e}}\left(\mathrm{t}_{\mathrm{M}}\right)$

The description of the proposed distribution strategy of the $C_{t o t}\left(t_{1}\right)$ credits among the various CSs is given in Sec. 3 . 
The life time of the assigned credits is equal to $d$, i.e. the duration of the signaling period. More precisely, with reference to the fig. 3 , credits assigned in the instant $t_{l}$ can be used by a CS within the interval $\left[t_{0}+\tau_{\max }, t_{0}+\tau_{\max }+d\right]$. The Mux only takes into account credits distributed in the last round trip time, i.e. in the last $\left\lceil\tau_{\text {max }} / d\right\rceil$ signaling cycles. This makes the control mechanism robust. Errors occurring during a signaling cycle are automatically recovered in the next cycles.

The release of the controlled mode and the transition to the uncontrolled mode takes place when the condition $b_{\text {mux }}^{e}\left(t_{M}\right)<T_{d}$ is satisfied. This event is communicated to the CSs via a dedicated signaling message (deactivation messages) instead of the credit assignment message.

The value of $T_{d}$ is fixed by imposing that: i) no cell loss occur at Mux; ii) the minimum time interval between the emissions of the deactivation and the successive activation messages must be not less than $d$. The second condition arises from the need to constrain the signaling rate below a fixed value known $a$ priori. These constraints can be matched by imposing that $T_{a}-T_{d}$ buffer positions are sufficient to store all the cells arriving at the Mux, in the worst case, during the interval $d-\tau_{\max }$. So

$\mathrm{T}_{\mathrm{d}}=\mathrm{T}_{\mathrm{a}}-\sum_{\mathrm{i}=1}^{\mathrm{N}}\left\lceil\frac{\mathrm{F}_{\mathrm{i}}}{\mathrm{L}_{\mathrm{c}}} \operatorname{Max}\left[0, \mathrm{~d}-\tau_{\max }\right]\right\rceil$

From (1) it arises that, if $d \leq \tau_{\max }, T_{d}=T_{a}$ that can be justified by considering that $T_{a}$ can not be exceeded before a time interval equal to $\tau_{\max }$, therefore the next activation can not take place before a time $d$. If $\mathrm{T}_{\mathrm{d}}<0$ the system will remain in the controlled mode.

The performance of the proposed ABR traffic control mechanism depend on the values of $\tau_{\max }$ and $d$. The strategy discussed for the evaluation of $b_{i}^{e}\left(t_{E}\right)$ and $b_{m u x}^{e}\left(t_{M}\right)$ well suits to access scenarios in which the value of $\tau_{\max }$ is low. If $\tau_{\max }$ increases, a loss of efficiency is expected. In this case, more sophisticated estimate procedures can be applied accepting cell loss at Mux [5]. As for the dimensioning of the signaling period $d$, it depends on the bandwidth that can be assigned to signaling. Such a choice is further influenced by the selected mode for conveying forward and backward signaling. The cell header (e.g. the GFC field) or the cell payload can be utilized for this scope. The former would allow a sensitive bandwidth saving but could imply a rough credit quantization [5].

\section{CREDIT DISTRIBUTION STRATEGY}

The credit distribution strategy here proposed, called Buffer Equalizing Distribution (BED) strategy, aims at equalizing, signaling period by signaling period, the occupancies of the transmission buffer of each CS. This strategy smoothes the differences among the buffer occupancies, providing a higher number of credits to those CSs whose buffers are approaching to overflow, and decreasing the credit rate to those CSs with large available buffer space. The operation of BED strategy can be summarized as follows.

Let $c_{i}\left(t_{1}\right)$ be the number of credits assigned to the $i$-th CS by the Mux at time $t_{1} ; c_{i}\left(t_{1}\right)$ is a non-negative integer and must satisfy the following constraints:

$\sum_{\mathrm{i}=1}^{\mathrm{N}} \mathrm{c}_{\mathrm{i}}\left(\mathrm{t}_{1}\right)=\mathrm{C}_{\mathrm{tot}}\left(\mathrm{t}_{1}\right)$

and

$0 \leq \mathrm{c}_{\mathrm{i}}\left(\mathrm{t}_{1}\right) \leq\left\lfloor\frac{\mathrm{P}_{\mathrm{i}}}{\mathrm{L}_{\mathrm{c}}} \mathrm{d}\right\rfloor$ 
The upper bound given by (3) specifies that the maximum number of credits that can be assigned to a CS can not exceed the maximum number of cells that can be emitted during a signaling period of duration $d$. Our problem is to assign the credits $c_{i}\left(t_{1}\right)$ so that all the CSs tend to have the same buffer occupancy.

To solve the above problem, let us suppose that the amount of credits assigned to the $i$-th $\mathrm{CS}$ at time $t_{l}$ can be a non-negative real number $c_{i}^{\prime}\left(t_{l}\right)$. The relation between the integer number $c_{i}\left(t_{1}\right)$ and the real number $c_{i}^{\prime}\left(t_{1}\right)$ will be given in the following.

By assuming that $K$ is a real number, representing the target buffer occupancy for all the CSs, the credit assignement follows the rule below:

$c_{i}^{\prime}\left(t_{1}\right)=\left\{\begin{array}{lll}0 & \text { if } & b_{i}^{e}\left(t_{E}\right)-K<0 \\ b_{i}^{e}\left(t_{E}\right)-K & \text { if } & 0 \leq b_{i}^{e}\left(t_{E}\right)-K \leq\left\lfloor\frac{P_{i}}{L_{c}} d\right\rfloor \\ \left\lfloor\frac{P_{i}}{L_{c}} d\right\rfloor & \text { if } & b_{i}^{e}\left(t_{E}\right)-K>\left\lfloor\frac{P_{i}}{L_{c}} d\right\rfloor\end{array}\right.$

The rule (4) aims at assigning to each CS as much credits as needed to achieve a transmission buffers occupancy equal to $K$ at time $t_{E}$. Consequently, if a CS is characterized by $b_{i}^{e}\left(t_{E}\right)<K$ the number of assigned credits will be null; conversely the third condition derives from (3).

The rule (4) can be syntetically expressed as:

$\mathrm{c}_{\mathrm{i}}{ }^{\prime}\left(\mathrm{t}_{1}\right)=\min \left\{\operatorname{Max}\left[0, \mathrm{~b}_{\mathrm{i}}^{\mathrm{e}}\left(\mathrm{t}_{\mathrm{E}}\right)-\mathrm{K}\right],\left\lfloor\frac{\mathrm{P}_{\mathrm{i}}}{\mathrm{L}_{\mathrm{c}}} \mathrm{d}\right\rfloor\right\}$

$1 \leq \mathrm{s} \leq \mathrm{N}$

The value of $\mathrm{K}$, maximizing the number of assigned credits, can be evaluated imposing that:

$\mathrm{N}$

$\sum_{i=1} c_{i}^{\prime}\left(t_{1}\right)=C_{t o t}\left(t_{1}\right)$

namely:

$\sum_{\mathrm{i}=1}^{\mathrm{N}} \min \left\{\operatorname{Max}\left[0, \mathrm{~b}_{\mathrm{i}}^{\mathrm{e}}\left(\mathrm{t}_{\mathrm{E}}\right)-\mathrm{K}\right],\left\lfloor\frac{\mathrm{P}_{\mathrm{i}}}{\mathrm{L}_{\mathrm{c}}} \mathrm{d}\right\rfloor\right\}=\mathrm{C}_{\mathrm{tot}}\left(\mathrm{t}_{1}\right)$

To solve the eq. (7) with respect to $K$, we define the function $G(K)$ as

$\mathrm{G}(\mathrm{K})=\sum_{\mathrm{i}=1}^{\mathrm{N}} \min \left\{\operatorname{Max}\left[0, \mathrm{~b}_{\mathrm{i}}^{\mathrm{e}_{\mathrm{E}}}\left(\mathrm{t}_{\mathrm{E}}\right)-\mathrm{K}\right],\left\lfloor\frac{\mathrm{P}_{\mathrm{i}}}{\mathrm{L}_{\mathrm{c}}} \mathrm{d}\right]\right\}-\mathrm{C}_{\mathrm{tot}}\left(\mathrm{t}_{1}\right)$

The solution of the equation $G(K)=0$ is the value of $K$ maximizing the number of assigned credits. As $G(K)$ is a monotonic function, not increasing with $K$, the solution can be easily determined by means of classical methods of numerical analysis (e.g. the Newton-like method). In our experience, the solutions are obtained in very few steps.

Once $c_{i}^{\prime}\left(t_{1}\right)$ has been found, the actual number of credits assigned to the $i$-th CS is:

$c_{i}\left(t_{1}\right)=$ Round $\left[c_{i}^{\prime}\left(t_{1}\right)+r_{i}\left(t_{1}-d\right)\right]$

wherein Round $[\mathrm{x}]=\lfloor\mathrm{x}+0.5\rfloor$ and $r_{i}\left(t_{1}-d\right)$ is the credit residual calculated in the last signalling cycle, according to

$r_{i}\left(t_{1}\right)= \begin{cases}0 & \text { if } t_{1}=t_{a}+\tau_{\max } \\ c_{i}^{\prime}\left(t_{1}\right)-c_{i}\left(t_{1}\right)+r_{i}\left(t_{1}-d\right) & \text { otherwise }\end{cases}$

wherein $t_{a}$ is the time in which the Mux starts the controlled mode. 
The credit residual $r_{i}\left(t_{1}\right)$ is a real number representing the sum of credit fractions unassigned to the $i$-th CS up to the time $t_{l}$. The value of $r_{i}\left(t_{1}\right)$ is not transferred to the source, but is stored by the Mux to be utilized in the next signaling period.

The application of relation (9) for the evaluation of the number of credits may involve that the constraint (2) is not satisfied. Therefore, a post-rearrangement of the credit assignment must be performed. In particular, if $\sum_{\mathrm{i}=1}^{N} \mathrm{c}_{\mathrm{i}}\left(\mathrm{t}_{\mathrm{E}}\right)<\mathrm{c}_{\text {tot }}\left(\mathrm{t}_{1}\right)$ more credits are distributed to those sources with higher $r_{i}\left(t_{E}\right)$, otherwise, a number of credits are subtracted to the sources with lower residuals.

The BED strategy allows a minimum number of transmission opportunities to be guaranteed also to CSs having very low emission rates. In fact, if $F_{\min }$ is the minimum bandwidth we want to guarantee to all sources, eq. (5) becomes:

$$
\mathrm{c}_{\mathrm{i}}^{\prime}\left(\mathrm{t}_{1}\right)=\min \left\{\operatorname{Max}\left[\frac{\mathrm{F}_{\min }}{\mathrm{L}_{\mathrm{c}}} \mathrm{d}, \mathrm{b}_{\mathrm{i}}^{\mathrm{e}}\left(\mathrm{t}_{\mathrm{E}}\right)-\mathrm{K}\right],\left\lfloor\frac{\mathrm{P}_{\mathrm{i}}}{\mathrm{L}_{\mathrm{c}}} \mathrm{d}\right]\right\} \quad 1 \leq \mathrm{i} \leq \mathrm{N}
$$

Summarizing, the evaluation of $c_{i}\left(t_{1}\right)$ takes place as follows:

1) Solve eq. (8) with $\mathrm{G}(\mathrm{K})=0$;

2) Evaluate $c_{i}^{\prime}\left(t_{1}\right)$ by using eq. (11);

3) Evaluate $\mathrm{c}_{\mathrm{i}}\left(\mathrm{t}_{1}\right)$ according to eq. (9);

4) Update $r_{i}\left(t_{1}\right)$ according to eq. (10).

As for the solution of eq. (8), a good starting point $K_{0}$ can be derived as follows.

We assume that, in eq. (4), $\mathrm{c}_{\mathrm{i}}^{\prime}\left(\mathrm{t}_{1}\right)=\mathrm{b}_{\mathrm{i}}^{\mathrm{e}}\left(\mathrm{t}_{\mathrm{E}}\right)-\mathrm{K}$ holds for every $i$. Summing and averaging eq. (4) for all the values of $i$, by taking into account eq. (5) we have:

$$
\mathrm{K}_{0}=\overline{\mathrm{b}}\left(\mathrm{t}_{\mathrm{E}}\right)-\overline{\mathrm{C}}
$$

wherein

$$
\overline{\mathrm{b}}\left(\mathrm{t}_{\mathrm{E}}\right)=\frac{\sum_{\mathrm{i}=1}^{\mathrm{N}} \mathrm{b}_{\mathrm{i}}^{\mathrm{e}_{\mathrm{e}}}\left(\mathrm{t}_{\mathrm{E}}\right)}{\mathrm{N}} \quad \text { and } \quad \overline{\mathrm{C}}=\frac{\mathrm{C}_{\mathrm{tot}\left(\mathrm{t}_{1}\right)}}{\mathrm{N}}
$$

It is to be observed that, if $G\left(K_{0}\right)=0, \mathrm{c}_{\mathrm{i}}\left(\mathrm{t}_{1}\right)$ would be equal to:

$$
c_{i}^{\prime}\left(t_{1}\right)=\bar{C}+b_{i}^{e}\left(t_{E}\right)-\bar{b}\left(t_{E}\right)
$$

The expression (14) synthetizes the credit assignment mode utilized by BED strategy. If $b_{i}^{e}\left(t_{E}\right)=\bar{b}\left(t_{E}\right)$, then the CSs will receive a number of credits equal to $\bar{C}$, i.e. equal to the value that would be assigned to it if the credits were equally distributed. Otherwise, if $b_{i}^{e}\left(t_{E}\right)>b\left(t_{E}\right)$ (or $\left.b_{i}^{e}\left(t_{E}\right)<b\left(t_{E}\right)\right)$, the source will receive a number of credits greater (or lower) than $\bar{C}$.

\section{PERFORMANCE STUDY}

Two homogeneous access scenarios have been distinguished, on the basis of the characteristics of the TSs emission process. As for the characteristics of the multiplexer, they are the same in both cases. In particular, in order to take into account the impact of the GB traffic, the Mux output capacity available for the ABR traffic has been modelled as an On-Off process. The Off periods correspond to the time intervals in which the Mux output capacity is dedicated to the 
GB traffic, and, consequently, the transmission of ABR cells is blocked. Moreover, the sources are placed at the same distance $l$ from the Mux. Obviously, $\tau_{\max }=2 l V_{p}$, wherein $V_{p}$ is equal to $5 \mu \mathrm{s} / \mathrm{km}$. The parameters of the multiplexer are reported in Table I.

In the following, the results relevant to the considered scenarios are described.

Table I Parameters of the simulation study

\begin{tabular}{lc}
\hline \multicolumn{1}{c}{ Parameter } & Value \\
\hline Source-Mux distance $(l)$ & $50 \mathrm{~km}$ \\
Mux buffer size $\left(\boldsymbol{B}_{\boldsymbol{m} u \boldsymbol{x}}\right)$ & $200 \mathrm{cells}$ \\
Number of sources $(N)$ & 14 \\
Mux outgoing capacity & $150 \mathrm{Mbit} / \mathrm{s}$ \\
Average Mux capacity available for ABR traffic $\left(F_{\text {mux }}\right)$ & $60 \mathrm{Mbit} / \mathrm{s}$ \\
Mean length of Mux Off periods & $10 \mathrm{cells}$ \\
Mean length of Mux On periods & $7 \mathrm{cells}$ \\
\hline
\end{tabular}

\subsection{On-Off sources}

First, the case in which all TSs emit according to an On-Off process, characterized by the same parameters values, has been simulated. The sources On and Off periods are distributed according to a truncated exponential distribution. The parameters describing the TSs are reported in Tab. II.

Table II Parameters of the TSs

\begin{tabular}{lc}
\hline \multicolumn{1}{c}{ Parameter } & Value \\
\hline TS average bit rate $(A)$ & $3.76 \mathrm{Mbit} / \mathrm{s}$ \\
TS peak bit rate $(F)$ & $20 \mathrm{Mbit} / \mathrm{s}$ \\
Mean length of TSs Off periods & $100 \mathrm{cells}$ \\
Maximum length of TSs On periods & $200 \mathrm{cells}$ \\
Transmission buffer size $(B)$ & $200 \mathrm{cells}$ \\
\hline
\end{tabular}

The credit-based mechanism is compared with the backpressure mechanism [2]. According to the backpressure, a CS is either in a uncontrolled or in a blocked mode. If a CS is in the uncontrolled mode, cells are sent to the Mux with rate $P=F$. In the blocked mode, emission of cells is denied. The transition from the uncontrolled to the blocked mode depends on the occupancy level of the Mux buffer. The blocking threshold $\left(T_{b}\right)$ has been fixed at

$\mathrm{T}_{\mathrm{b}}=\mathrm{B}_{\text {mux }}-\mathrm{N}\left\lceil\frac{\mathrm{P}}{\mathrm{L}_{\mathrm{c}}} \tau_{\max }\right\rceil$

The reverse transition occurs when the buffer occupancy returns below $T_{u}=T_{b}-N$.

Two values of $P(P=20 \mathrm{Mbit} / \mathrm{s}$ and $150 \mathrm{Mbit} / \mathrm{s})$ have been considered for the credit-based mechanism, whereas $P=20 \mathrm{Mbit} / \mathrm{s}$ has been assumed for the backpressure . In fact, higher values would lead to a decrease of the maximum value of the access latency tolerated by the mechanism. From this choice the maximum value of the source-mux distance equals $l=28 \mathrm{~km}$.

In Figure 3, the cell loss probability vs. the offered load normalized with respect to $F_{\text {mux }}$ is plotted. The backpressure mechanism is compared with the credit mechanism. For this last one, both the cases of CS peak rate equal to $150 \mathrm{Mbit} / \mathrm{s}$ and $20 \mathrm{Mbit} / \mathrm{s}$ are plotted. The traffic variation is obtained by changing the number of active sources. It can be noted that the CS peak bit rate slightly influences the cell loss probability. Moreover, close to the saturation conditions, i.e. for loads approaching to 1 , the performance is independent of the used control mechanism, while for lower loads the advantages provided by the credit based mechanism are sensitive. 
In the figure the $95 \%$ confidence intervals of the obtained results are also plotted; confidence intervals of the same order of magnitude have been obtained for all the graphs in this section.

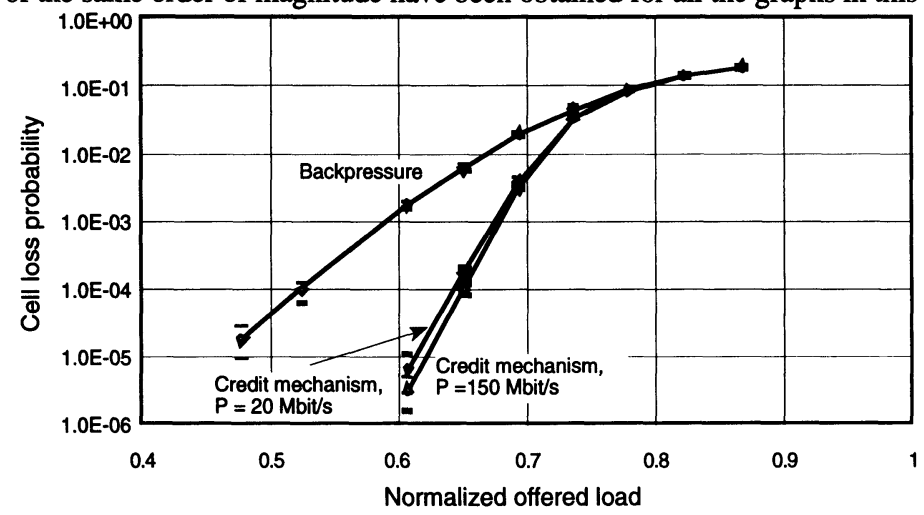

Figure 3 - Cell loss probability vs. offered load.

The Figure 4 shows the cell loss probability of the credit-based mechanism vs. $l$. The number of sources is equal to 14 , corresponding to an average load normalized with respect to $F_{m u x}$ equal to about 0.877 . The loss probability is plotted for various values of $d$, and for $P=150 \mathrm{Mbit} / \mathrm{s}$ only. The loss probability for the credit-based mechanism is compared with that relevant to the backpressure mechanism and with the lower bound calculated as follows.

For high values of latency the throughput performance is limited by the maximum number of credits that can be sent by the Mux in $\tau_{\max }$ (i.e. $B_{m u x}$ ). The maximum throughput $T H_{\max }$ is given by $\mathrm{TH}_{\max }=\frac{\mathrm{B}_{\operatorname{mux}} \cdot \mathrm{L}_{\mathrm{c}}}{\tau_{\max }}$. By supposing that all the $N$ TSs have the same average bit rate $A$ and $\tau_{\max }>\frac{B_{\operatorname{mux}} \cdot L_{c}}{N \cdot A}$, a lower bound of the cell loss probability is given by:

$\pi_{\text {loss }}=1-\frac{\mathrm{B}_{\operatorname{mux}} \cdot \mathrm{L}_{\mathrm{c}}}{\mathrm{N} \cdot \mathrm{A} \cdot \tau_{\max }}$

Eq. (16) is independent of the particular distribution of the source emission process.

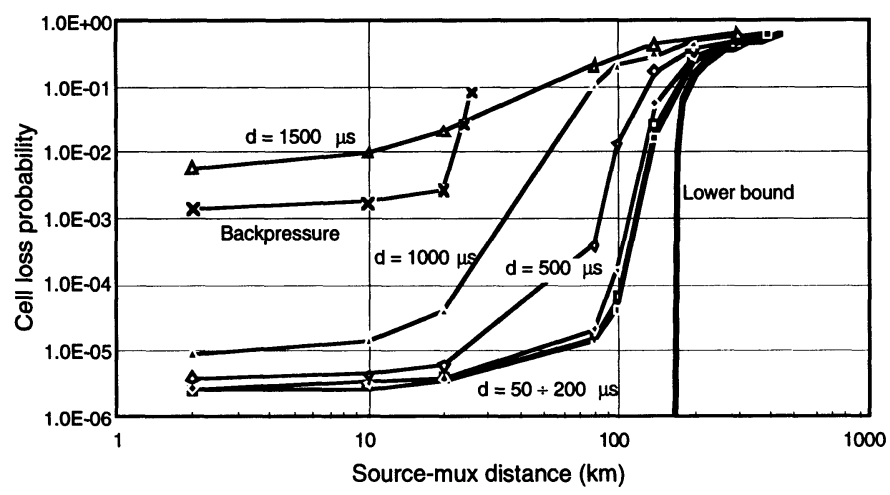

Figure 4 - Cell loss probability vs. source-multiplexer distance 
From Figure 4, it arises that, at low latency, both mechanisms present a first zone where performance is almost independent of $l$. It is also evident that in this zone the performance of the credit based mechanism is quite better than that of the backpressure. A further increase of $l$ corresponds to a sharp performance worsening. For the credit based mechanism this zone is roughly located at a latency value being 6 times that relevant to the backpressure. Finally, for high values of $l$ the performance of the credit based mechanism is close to the lower bound given by (16). That implies that the behaviour of the credit-based mechanism here proposed can be considered as almost ideal. Better performance can be achieved only if loss at the Mux is tolerated [5].

Finally, Figure 5 plots the cell loss probability for the credit based mechanism vs. the signaling period $d$, for different values of latency. The figure shows that, in a wide range of values of $d$, this parameter has a slight impact on the loss performance.

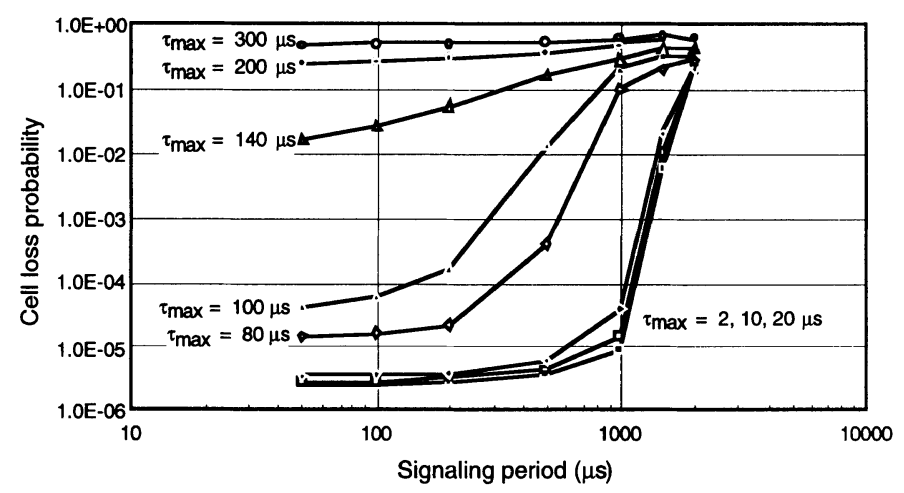

Figure 5 - Cell loss probability vs. signaling period

Finally, a set of trials has been carried out to investigate the fairness of the credit-based mechanism. The same parameters of Tables I and II have been adopted, except for $F_{m u x}=50$ $\mathrm{Mbit} / \mathrm{s}$. As for the source mean bit rate, two sets of sources have been distinguished in each trial, such that the average rate offered to the source is $\mathrm{N} \cdot \mathrm{A}$ Mbit/s. The Table III reports the cell loss probability for the two sets of sources both for the credit-based mechanism and the backpressure. In the case indicated with " $x-y$ " a set of seven sources transmit with a mean bit rate of $x \mathrm{Mbit} / \mathrm{s}$, while the remaining seven sources transmit with a mean bit rate of $y \mathrm{Mbit} / \mathrm{s}$.

Table III Cell loss probability for different sources

\begin{tabular}{cccccccc}
\hline \multicolumn{3}{c}{ Credit-based mechanism } & \multicolumn{3}{c}{ Backpressure mechanism } \\
\hline Case & Low & High & Mean & Case & Low & High & Mean \\
\hline $4-4$ & & & 0.0216 & $4-4$ & & & 0.0390 \\
\hline $3-5$ & 0.0220 & 0.0223 & 0.0222 & $3-5$ & 0.0185 & 0.0535 & 0.0404 \\
\hline $2-6$ & 0.0220 & 0.0233 & 0.0229 & $2-6$ & 0.0059 & 0.0536 & 0.0418 \\
\hline $1-7$ & 0.0201 & 0.0259 & 0.0251 & $1-7$ & 0.0013 & 0.0485 & 0.0427 \\
\hline $0-8$ & & 0.0322 & 0.0322 & $0-8$ & & 0.0420 & 0.0420 \\
\hline
\end{tabular}

As it can be noted, the loss probability increases as the traffic unbalancement grows for both the credit-based mechanism and the backpressure. However, the table shows that, in the case of the backpressure, the difference in the cell loss probability experienced by the two load classes is much more different. This is due to the BED credit distribution strategy adopted in the credit-based mechanism, which advantages stations close to loss conditions. 


\subsection{LAN sources}

For the second access scenarios data arising from LAN traffic measurements have been employed. In particular, the files provided by Bellcore reporting the actual traffic measurement on an Ethernet cable at the Bellcore Morristown Research and Engeneering facility, and relevant to the periods of August and October 1989 have been utilized. The data traces have been divided in 14 adjacent time intervals, each representing the packet arrival process for a TS in a fixed time interval. The data in the files have been analyzed in order to obtain the source parameters. It results that each source is characterized by a peak bit rate of appx. $10 \mathrm{Mbit} / \mathrm{s}$. Figure 6 plots the histogram of the packet length relevant to the October file. Peaks corresponding to the minimum and maximum packet size equal to 64 and 1518 bytes, respectively, can be noticed. The histogram of the packet length relevant to the August file is very similar to this one. As far as the average bit rate, it equals approximately $3.33 \mathrm{Mbit} / \mathrm{s}$ for the October file, while it equals approximately $1.29 \mathrm{Mbit} / \mathrm{s}$ for the August file. Finally, the source buffers are 200 cells wide.

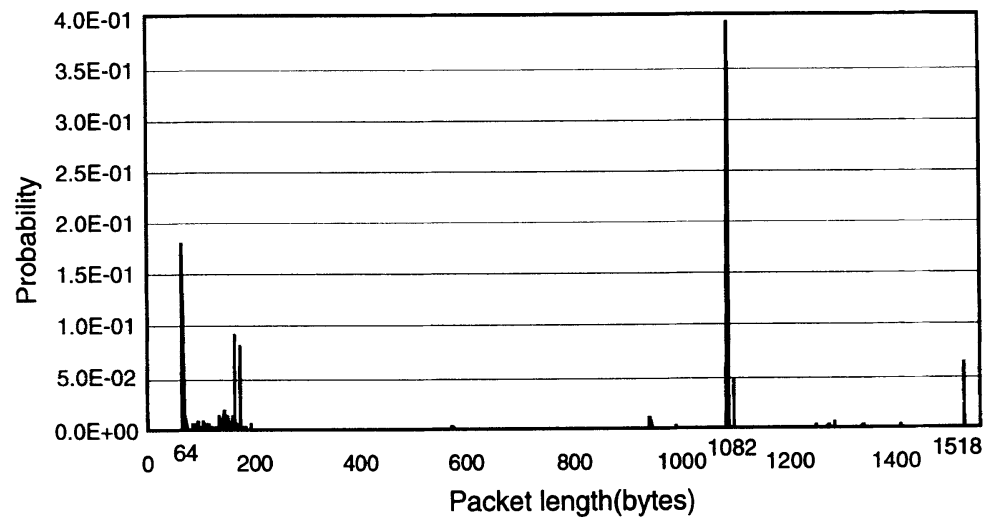

Figure 6 - Histogram of the packet length.

The Figure 7 plots the cell loss ratio for both the credit and the backpressure mechanism vs. the available bandwidth at the multiplexer output. The Mux parameters are those of Table II.

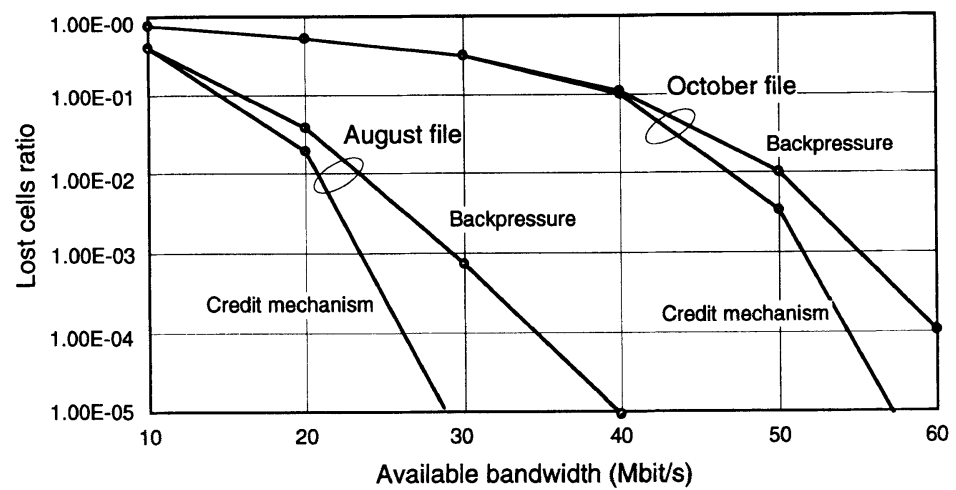

Figure 7 - Cell loss probability for the LAN sources. 
The figure plots the results relevant to both the August and October files. Since the available data set is limited, very low values of cell loss ratio cannot be estimated, and the variance of results is much higher than in the On-Off case. Obviously, in the case of the August traces the cell loss ratio is considarably lower; however, in both cases the credit mechanism performs orders of magnitude better than the backpressure in the low loss region.

\section{REFERENCES}

[1] P. Newmann: "Backward Explicit Congestion Notification for ATM Local Area Networks", Proc. Globecom'93, Houston, 29 November - 2 December 1993.

[2] H.T. Kung, A. Chapman: "The FCVC (Flow-Controlled Virtual Channels) Proposal for ATM Networks: A Summary" Proc. 1993 Internation Conf. on Network Protocols, San Francisco, California, 19-22 October 1993.

[3] J. Cherbonnier, J.Y. Le Boudec: "A GFC protocol for congestion avoidance in the ATM connectionless service"; Proceedings of EFOC/LAN '92, Paris.

[4] G. Bagnoli, M. Listanti, R. Winkler: "Cell level and frame level performance of traffic control schemes for no resource reservation data communications in ATM networks"; Proceedings of the 14th International Teletraffic Congress - ITC'94, Antibes, France, 6-10 June 1994.

[5] G. Chierchia: "Analysis of credit based mechanisms for no bandwidth reservation traffic control"; Thesis on electronic engineering, University of Roma "La Sapienza"; in Italian.

\section{BIOGRAPHIES}

Francesco Bernabei graduated in Electronic Engineering at the University of Rome "La Sapienza" in 1984. After a one year University grant, he joined, in 1985, Fondazione Ugo Bordoni, where he is currently the leader of the "Telecom Network Architectures" group. He has been involved in the following research areas: modelling, simulation and analysis of ATM switching architectures, LAN/MAN architectures, traffic and congestion control. He is a member of IEEE Communications and Computer Society and a member of ACM.

Gianluca Chierchia received his Dr. Eng. degree in Electronics Engineering in 1994 from the University of Roma "La Sapienza". His main research interests are in the area of traffic control in ATM networks.

Laura Gratta received her Dr. Eng. degree in Electronics Engineering in 1989 from the University of Roma "La Sapienza". In 1990 she joined the Fondazione Ugo Bordoni in the Networks and Services division, where she currently works as a researcher. Her main research interests are in the areas of modelling, simulation and analysis of Gigabit networks and traffic control in ATM networks. Dr. Gratta is a member of IEEE and INFORMS.

Marco Listanti received his Dr. Eng. degree in Electronics Engineering from the University "La Sapienza" of Roma in 1980. He joined the Fondazione Ugo Bordoni in 1981, where has been leader of the group "TLC network architecture" until 1991. In November 1991 he joined the INFOCOM Dept. of the University of Roma "La Sapienza", where he is Associate Professor in Switching Systems. His current research interests focus on ATM networks with particular regard to traffic control and signalling aspects. Prof. Listanti is also a member of IEEE Communications and Computer Societies. 